\title{
Does pulmonary metastasectomy of colorectal metastases translate to better survival? A systematic review
}

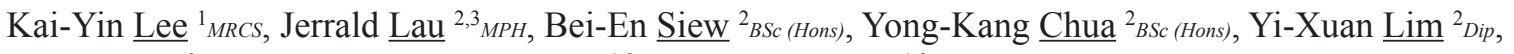
Xin-Yi Lim ${ }^{3}{ }_{B S C \text { (Hons) }}$, Choon-Seng $\underline{\text { Chong }}{ }^{1,2}{ }_{\text {FRCS }}$, Ker-Kan Tan ${ }^{1,2}$ PhD

\begin{abstract}
Introduction: Surgical resection of the primary and metastatic tumour is increasingly recommended in suitable patients with metastatic colorectal cancer (CRC). While the role of metastasectomy is well studied and established in colorectal liver metastasis, evidence remains limited in pulmonary metastases. This systematic review was conducted to examine the current evidence on the role of lung metastasectomy (LUM) in CRC.

Methods: Three databases were systematically searched, to identify studies that compared survival outcomes of LUM, and factors that affected decision for LUM.

Results: From a total of 5,477 records, 6 studies were eventually identified. Two papers reported findings from one randomised controlled trial and 4 were retrospective reviews. There was no clear survival benefit in patients who underwent LUM compared to those who did not. When compared against patients who underwent liver metastasectomy, there was also no clear survival benefit. Patients who underwent LUM were also more likely to have a single pulmonary tumour, and metachronous disease.

Conclusion: The evidence suggests a role for LUM, but is limited by inherent selection bias in retrospective reviews, and the single randomised clinical trial performed was not completed. More prospective studies are required to understand the true effect of LUM on outcomes in metastatic CRC.
\end{abstract}

Ann Acad Med Singap 2021;50:773-81

Keywords: Colorectal cancer, pulmonary metastasectomy, pulmonary metastases, survival

\section{INTRODUCTION}

Colorectal cancer (CRC) is the one of most common cancers worldwide. Metastatic disease occurs in approximately half of all CRC patients, in either synchronous or metachronous presentations. ${ }^{1}$ These patients form a heterogenous group that vary in presentation, disease progression and treatment options.

Advancements in surgery and chemotherapy have revolutionised the management of metastatic CRC. In addition to chemotherapy, surgical clearance of all disease, whether primary or metastatic, is often recommended in suitable patients. Reported 5-year survival after R0 resection of primary and metastatic tumours in metastatic CRC ranges from 40.0 to $58.0 \%$, compared to just 3.0 to $5.0 \%$ without any treatment. ${ }^{2-5}$
While the role of liver metastasectomy or utilisation of other adjuncts to address colorectal liver metastases has increasingly been advocated, many are now debating if such a similar approach can be advocated in patients with lung metastases from CRC. Although slightly less common, pulmonary metastases (PM) still occur in $10.0-20.0 \%$ of patients with CRC, with approximately half of these patients presenting with synchronous metastases. ${ }^{6,7}$ Patients with PM are now being considered for curative metastasectomy if both the primary CRC and PM can be resected with clear margins and if they are fit. ${ }^{6}$ In spite of the burgeoning literature looking at liver metastasectomy (LIM), limited evidence for lung metastasectomy (LUM) exists with a majority of them being case series and retrospective studies that

\footnotetext{
${ }^{1}$ University Surgical Cluster, National University Health System, Singapore

${ }^{2}$ Yong Loo Lin School of Medicine, National University of Singapore, Singapore

${ }^{3}$ Saw Swee Hock School of Public Health, National University of Singapore, Singapore

Correspondence: A/Prof Ker-Kan Tan, Department of Surgery, Yong Loo Lin School of Medicine, National University of Singapore, NUHS Tower Block, Level 8, 1E Kent Ridge Road, Singapore 119228.

Email:surtkk@nus.edu.sg
} 


\section{CLINICAL IMPACT}

\section{What is New}

- There was no clear survival benefit in patients who underwent lung metastasectomy (LUM) compared to those who did not.

- Evidence suggests a role for LUM, but is limited by inherent selection bias.

\section{Clinical Implications}

- Role of metastasectomy remains limited in pulmonary metastases.

- More prospective studies are required to understand the true effect of LUM on outcomes in metastatic colorectal cancer.

reported acceptable survival rates. ${ }^{6,8}$ However, there are currently no guidelines on the role of resection of pulmonary metastases from CRC. This review aims to examine the current literature and evidence on the role of pulmonary metastasectomy in colorectal cancer.

\section{METHODS}

\section{Search strategy and selection criteria}

A systematic search of three databases (PubMed, Embase, and CENTRAL) was conducted on 5 June 2020. The search strategy aimed to capture terms that were relevant to pulmonary metastases, LUM and colorectal cancer. The search strategy was kept deliberately broad to mitigate the possibility of relevant articles being missed out.

Terms searched were: ("pulmonary metastasectomy" OR "lung metastasectomy" OR "surgery" OR "resection") AND ("pulmonary metastases" OR "pulmonary metastasis" OR "lung metastases" OR "lung metastasis") AND ("colorectal cancer" OR "colon cancer" OR "colorectal").

We included all studies that reported survival as a primary outcome of surgical resection of a colorectal pulmonary metastasis that were published in English. Studies were excluded if they (1) did not collect primary data (e.g. reviews, meta-analyses or commentaries), (2) did not include any comparators (i.e. case series), (3) enrolled patients with a non-colorectal primary cancer site, (4) did not involve any form of surgical treatment, and (5) focused specifically on reporting surgical techniques.
We also excluded studies that (6) only enrolled specific subgroups of the patient population of interest (e.g. only repeat LIM or LUM, or simultaneous liver and lung metastases) due to the potential for these subgroup disease and treatment characteristics to confound the overall impact of LUM.

\section{Study selection, data extraction, and analysis}

The search strategy was applied to each of the 3 databases and 1 co-author (BES) compiled the resultant records using EndNote X8. These were subjected to a preliminary screening of titles and abstracts by 4 co-authors (BES, YKC, LYX and YXL) using the study selection criteria detailed above. The full texts of these shortlisted records were then reviewed by 4 co-authors (JL, BES, YKC and YXL). To mitigate possible selection biases, $10 \%$ of each co-author's assigned full texts were independently reviewed by another co-author, and any disagreements were resolved via consensus among all authors.

Quality assessment and risk of bias were performed for each included study by two co-authors (BES and $\mathrm{JL}$ ) using the appropriate Joanna Briggs Institute (JBI) critical appraisal tools checklist (based on the study design). ${ }^{9}$ Data extraction for the finalised sample of studies included was performed by 1 co-author (BES) using a standardised electronic data collection form. We then descriptively summarised study and sample characteristics, key findings and limitations from each of the included studies.

\section{RESULTS}

The search yielded a total of 5,477 records (1,472 from PubMed, 3,926 from Embase and 79 from CENTRAL), of which 1,232 duplicates were removed. Of the remaining 4,245 records, 4,049 were excluded after preliminary screening of titles and abstracts based on our study selection criteria. The remaining 196 records were subjected to a full text review, resulting in a final sample of 6 articles. The PRISMA flow chart illustrating the search and study selection process can be found in Fig. 1. These 6 studies satisfied more than $50 \%$ of the JBI critical appraisal criteria, and were deemed to be of acceptable quality. The studies are summarised in Table 1, describing the year of publication, country of origin, sample size, study design, disease-free survival (DFS), overall survival (OS), interval to LUM from diagnosis, number of lung metastases, concurrent liver and lung metastases, and chemotherapy. 


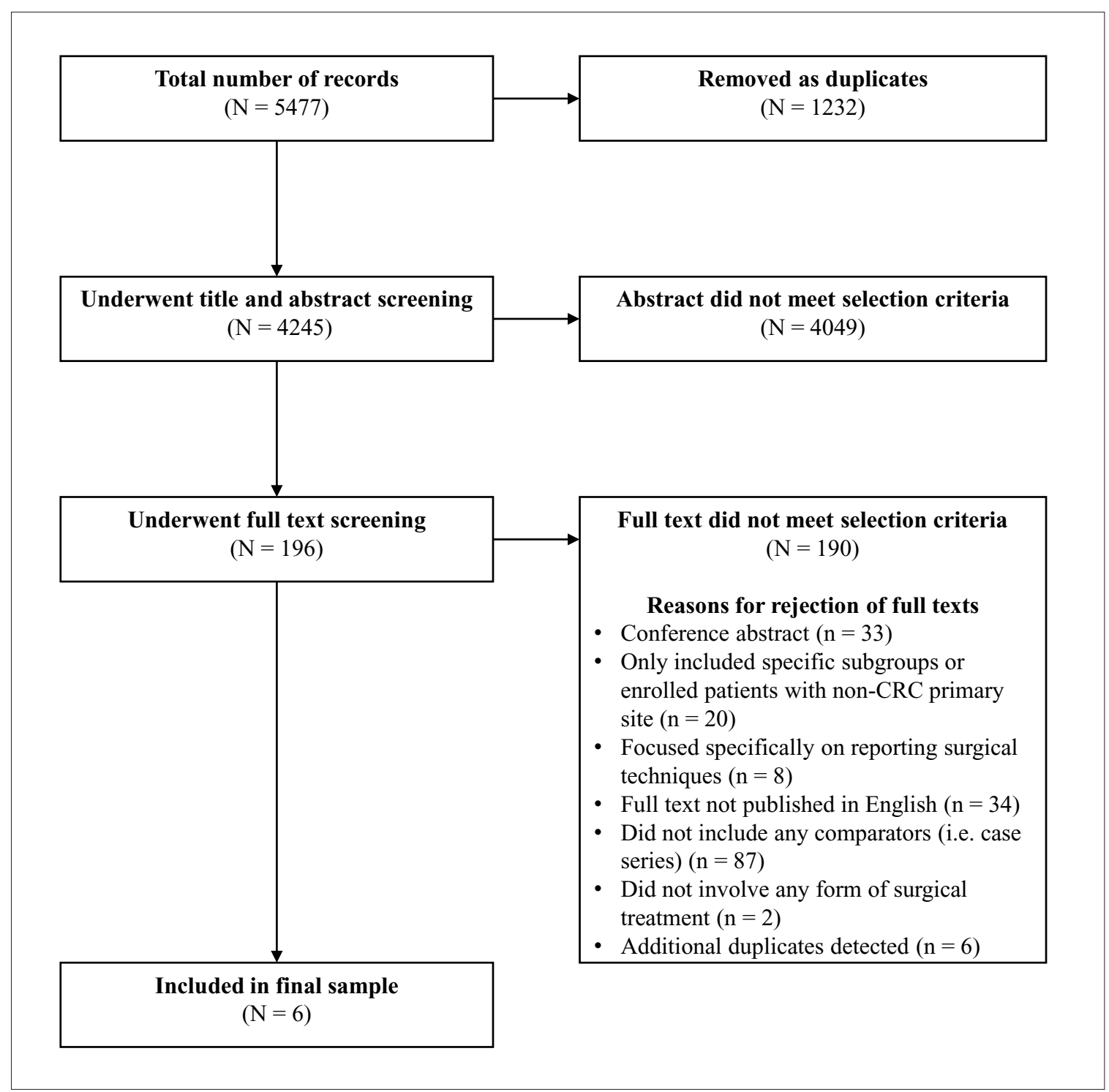

Fig. 1. PRISMA flowchart of article selection process. CRC: colorectal cancer

\section{Descriptive characteristics}

Out of the 6 studies, 2 were randomised controlled trials (RCTs) and the remaining 4 were retrospective cohort studies. ${ }^{10,11}$ The 2 RCTs were based on the same database, with Milosevic et al. adding 28 more patients to the original dataset. ${ }^{11}$ In terms of comparators, 3 studies compared patients who underwent LUM to patients who did not, two studies compared patients who underwent LUM to LIM, and 1 study analysed both comparators. The sample sizes in the studies were mostly small, with the exception of Zong et al. who studied 1047 patients with CRC LUM. ${ }^{12}$ With the exception of Iwasaki et al. who published their study in 2005 , the publication dates were more recent for the rest of the included sample, ranging from 2015 to 2020 .

\section{No clear benefit in overall survival with LUM compared to no LUM}

There were 4 studies that compared patients who underwent LUM versus no surgery, of which 2 were RCTs and the other 2 retrospective cohort studies. While the RCTs reported 5-year overall survival (OS), the retrospective studies analysed median OS. The RCTs included multiple variables for minimisation including CRC stage, prior liver resection, interval to surgery from diagnosis, and number of PM, creating balanced groups for randomisation. Both studies reported similar OS after LUM, with Treasure et al. reporting an estimated 5 -year OS of $38.0 \%$ (95\% confidence interval [CI] 23-62) for LUM group vs $29.0 \%$ (95\% CI 16-52) in the control group. ${ }^{10}$ Milosevic et al. reported an estimated 


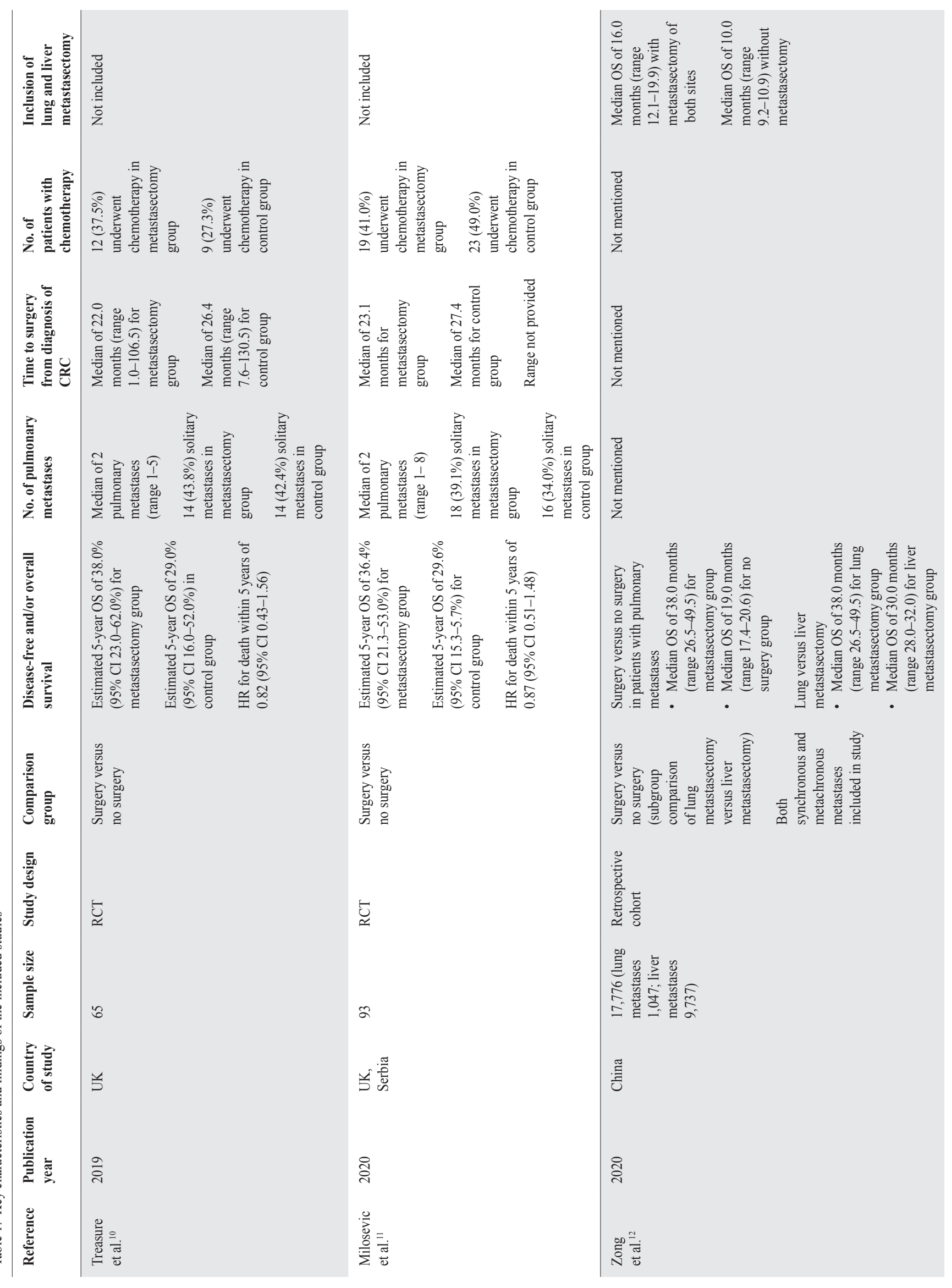




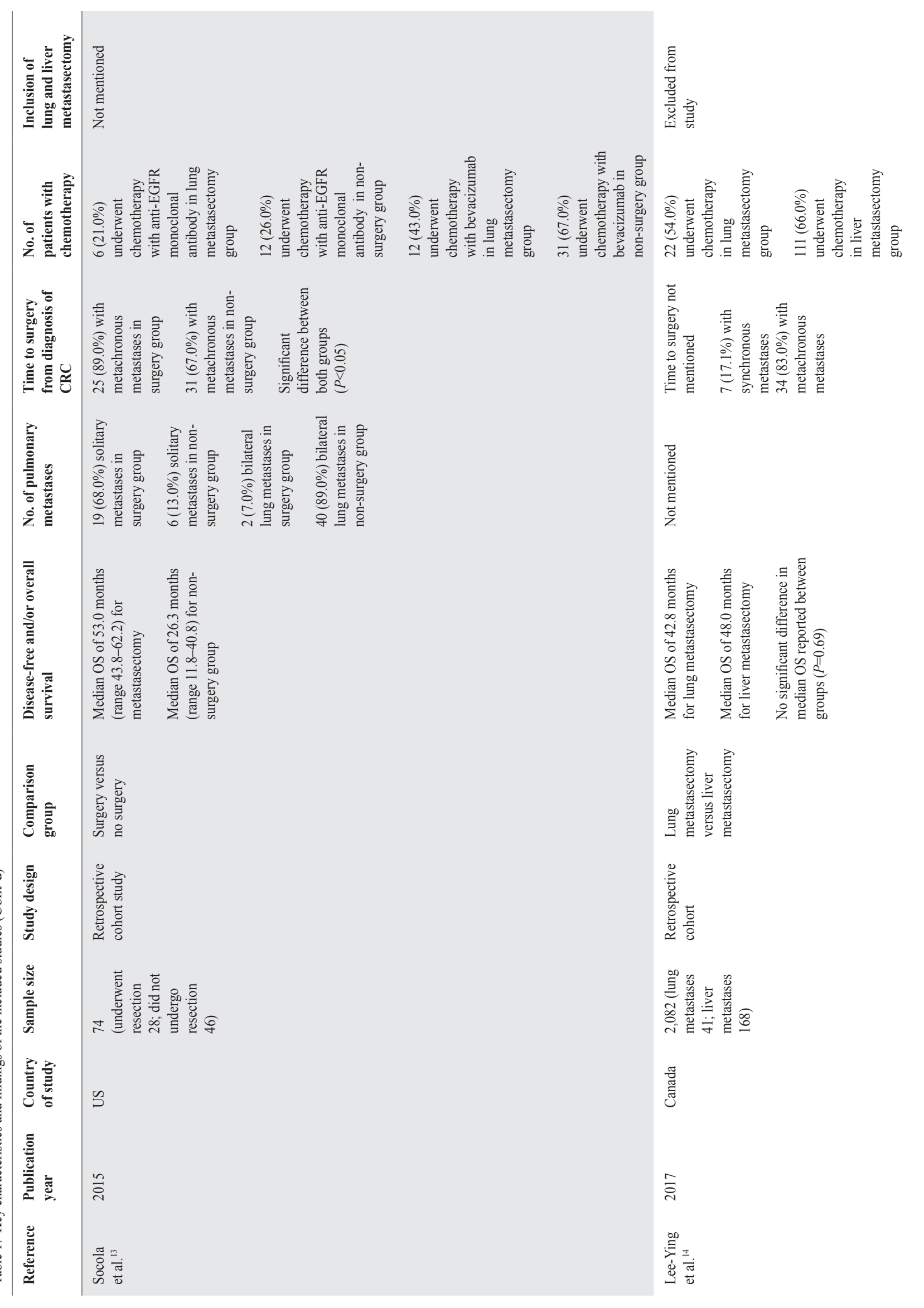




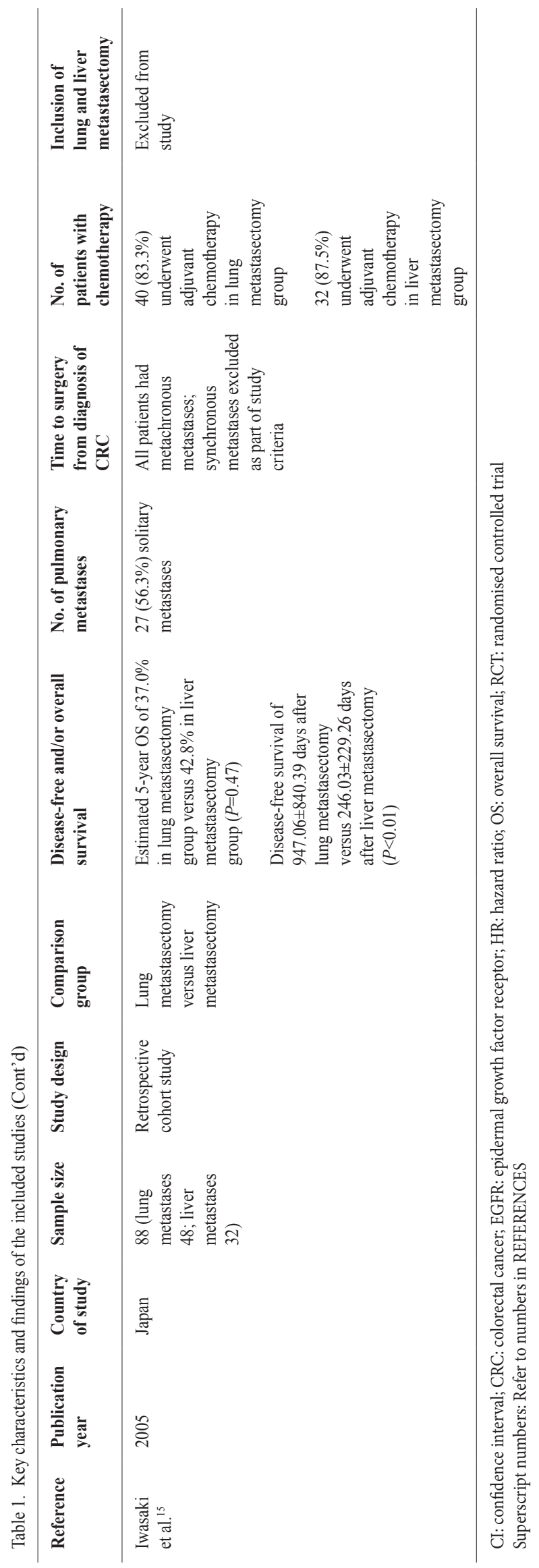

5-year OS of $36.4 \%(95 \%$ CI $21.3-53.0)$ for LUM group vs $29.6 \%$ (95\% CI 15.3-45.7) in the control group. ${ }^{1}$ The difference in OS with or without LUM was not statistically significant.

In the retrospective studies, Zong et al. reported median OS of 38 months (range 26.5-49.5) for LUM group vs 19 months (range 17.4-20.6) for patients who did not undergo surgery. ${ }^{12}$ Socola et al. had better median OS of 53 months (range 43.8-62.2) for LUM group vs 26.3 months (range 11.8-40.8) for patients who did not undergo surgery. ${ }^{13}$ Similarly, the difference in median OS were not statistically significant.

\section{LUM is not associated with better overall survival} compared to LIM

There is conflicting evidence in survival outcomes after LUM compared to LIM. Only retrospective data was available in this comparison. Out of the 3 studies, two showed worse OS in patients who undergo LUM, although they were not shown to be statistically significant. ${ }^{2-4}$ Lee-Ying et al. reported median OS of 42.8 months after LUM compared to 48.0 months after LIM $(P=0.69){ }^{12,14,15}$ Similarly, Iwasaki et al. reported estimated 5 -year OS of $37.0 \%$ after LUM vs $42.8 \%$ after LIM $(P=0.46) .{ }^{14}$ However, Zong et al. reported better survival after LUM, with a median OS of 38 months (range 26.5-49.5) after LUM versus 30 months (range 28.0-32.0) after LIM. ${ }^{12}$

\section{LUM is associated with longer disease-free survival than LIM}

Only 1 study, Iwasaki et al., reported disease-free survival (DFS). When compared to LIM, LUM was associated with significantly longer median DFS (947.06 \pm 840.39 versus $246.03 \pm 229.26$ days, $P<0.01) .{ }^{15}$ Additional LUM for the patients who developed recurrence was not reported.

\section{Patients who underwent LUM were more likely to have unilateral and solitary PM}

Both RCTs included the number of PM in variables used for minimisation. They included $43.8 \%$ and $39.1 \%$ versus $42.4 \%$ and $34.0 \%$ of patients with solitary PM in the LUM and control group, respectively. The median number of metastases was 2 , and ranged from 1 to 8 metastases.

Only 2 of the retrospective studies reported the number of PM in patients. In Socola et al., there were more patients with solitary PM that underwent LUM compared to those who did not (68.0\% vs $13.0 \%) .{ }^{13}$ No statistical analysis was provided. More patients (56.3\%) who underwent LUM had solitary PM in the study by 
Iwasaki et al. Only 1 study reported the incidence of bilateral PM. ${ }^{13}$ Majority of patients who did not undergo surgery had bilateral PM (89.0\% vs 7.0\%). However, subsequent univariate analysis of prognostic factors in patients who underwent resection did not show any significant association of bilateral PM with survival $(P=0.717)$.

\section{Majority of patients who undergo LUM had metachronous PM}

Time to LUM from diagnosis of CRC was not well reported in most studies. Majority of patients studied were those with metachronous PM. One study excluded patients with synchronous PM. ${ }^{15}$ In patients who undergo LUM, majority present with metachronous PM. ${ }^{13,14}$ Socola et al. also reported that significantly more patients with metachronous PM undergo LUM $(89.0 \%$ versus $67.0 \%, P<0.05) .{ }^{13}$

For Treasure et al. and Milosevic et al., the median time to randomisation from diagnosis of CRC was also included into variables used for minimisation. ${ }^{10,11}$ While the metastasectomy group had a shorter median duration by $4.3-4.4$ months, this was not significant (22.0 and 23.1 months versus 26.4 and 27.4 months). However, it is worth noting that the shortest interval in the LUM group in Treasure et al. was 1 month. ${ }^{10}$

\section{Large variation in uptake and regimens of postoperative chemotherapy for $\mathbf{P M}$}

The uptake of postoperative chemotherapy and types of chemotherapy regimens after metastasectomy differs widely between studies. There were 5 studies that reported on chemotherapy treatments. Uptake for postoperative chemotherapy was low in Treasure et al., with fewer patients in the control group undergoing chemotherapy $(27.3 \%$ versus $37.5 \%) .{ }^{10}$ Milosevic et al. reported higher postoperative chemotherapy rates overall, with more patients undergoing chemotherapy in the control group (41.0\% versus $49.0 \%) .{ }^{11}$ In 1 study, patients with more than Dukes A CRC were administered postoperative chemotherapy consisting tegaful and uracil (UFT) with leucovorin. ${ }^{15}$ Majority of patients underwent this postoperative therapy in both LUM and LIM groups (83.3\% and $87.5 \%$ respectively). Lee-Ying et al. reported postoperative chemotherapy in $54.0 \%$ of patients with LUM and a slightly higher rate of $66.0 \%$ in patients who underwent LIM. ${ }^{14}$ While Socola et al. did not report actual proportion of patients who underwent chemotherapy, it was shown that bevacizumab was the most commonly used drug in both groups: $n=12(43.0 \%)$ and $n=31(67.0 \%)$ in LUM and no LUM group, respectively. ${ }^{13}$
The use of radiation therapy (RT) or radiofrequency ablation (RFA) in the treatment of PM was only described in the 2 RCTs. ${ }^{10,11}$ Only a few patients (range 9-11) received RT during the 5-year follow-up period, of which half of the patients were from the control group. However, these numbers included RT for other locations of metastasis such as brain and bone. ${ }^{10,11}$ The use of RFA was also very limited in these studies, and only 2-3 patients received RFA in both the control and LUM group ${ }^{10,11}$ within 5 years of follow-up.

\section{DISCUSSION}

Pulmonary metastasectomy is regularly performed for a significant proportion of patients with metastatic CRC with PM, but is still lacking in quality evidence. Gonzalez et al. published a meta-analysis in 2013 looking at risk factors that affect survival after LUM, but only included retrospective single-arm studies. ${ }^{16}$ In our study, we identified at least 195 studies about PM and LUM. Majority of the studies were removed due to the lack of comparators (e.g. case series). Out of the 6 remaining studies, 2 were based on the same RCT (PulMiCC), which was terminated early due to poor outcomes in the treatment group and worsening recruitment.

From our results, LUM does not show clear survival benefit at present. Studies that compared LUM against no surgery showed that patients who undergo LUM had better OS, although not statistically significant. The reported 5-year survival from the 2 RCTs was $36.0-38.0 \%$, which was slightly lower than earlier reported 5-year survival of $40.0-60.0 \%$ based on case series and single-arm retrospective studies. ${ }^{10,11}$ However, those who did not undergo surgery still had a 5 -year OS of $29 \%$, which is better than previously reported. ${ }^{5}$ When compared against patients who undergo LIM, the results are less consistent. Two studies reported worse survival outcomes with LUM compared to LIM while another study reported otherwise; however, the results were not statistically significant. Even so, the reported survival outcomes for both LUM and LIM are better than those who do not undergo surgery. The reported 5-year survival of $37.0 \%$ after LUM is consistent with other studies. ${ }^{10,11,15-17}$ Disease-free survival was only reported in a single study, which showed significantly better DFS with LUM. The time to recurrence was reported to be almost 3 times longer with LUM compared to LIM. This differs from several other large retrospective studies, which reported recurrence rates in LUM similar to that of LIM, with reported recurrence of up to $70.0-80.0 \%$ at 2 years. ${ }^{18-20}$ 
We also looked at several factors that may affect decision for LUM. In retrospective studies, patients with unilateral and solitary PM were more likely to undergo LUM. Only $7.0 \%$ of patients with bilateral PM underwent surgery in 1 study. ${ }^{13}$ Other prognostic factors such as LUM resection margins and presence of hilar or mediastinal nodes were not available in these studies.

Majority of patients who undergo LUM also had metachronous metastases, which is consistent with other retrospective reviews that reflect better survival outcomes in patients who present with metachronous PM. ${ }^{6,710,21}$ Survival data based on number of PM and metachronous presentation were not available based on these few reviews. In the meta-analysis by Gonzalez et al., shorter DFS was associated with increased mortality (hazard ratio [HR] 1.59, 95\% CI $1.27-$ 1.98). Similarly, multiple PM was associated with increased mortality (HR 2.04, 95\% CI 1.72-2.41). ${ }^{16}$ Chemotherapy is the main treatment for metastatic CRC, but uptake of adjuvant chemotherapy remains variable, ranging from 37.5-87.5\%..$^{10,11,13,14}$ Reasons for no postoperative chemotherapy were not provided, and prior chemotherapy treatment were not available. There are currently no guidelines available on the duration of chemotherapy specifically for postmetastasectomy and would be an important aspect to address in the overall treatment of metastatic CRC.

Overall, the evidence suggests that there appears to be a role for LUM, but is limited by the quality of research available. Four out of 6 studies were retrospective in nature, with strong selection biases in decision for metastasectomy. Metastatic disease in CRC encompasses a spectrum of patients, and those who undergo LUM are likely to have more favourable patient and disease prognostic factors. Patients who are not fit to undergo chemotherapy or surgery, synchronous presentation, bilateral pulmonary tumours or multiple sites of metastases are less likely to undergo LUM. This selection bias was perhaps compounded by the lack of controlling for other confounders, including molecular status (such as BRAF, RAS and microsatellite instability).

While RCTs are ideal to overcome selection biasthe PulMiCC trial did include key variables for minimisation to allow for balanced groups to be studied - only 2 of the included studies in this review were RCTs, and both reflect the challenges of limited sample size in conducting a trial in such a complex group of patients. Of the 512 patients that were recruited at the first stage of the trial, 419 patients did not undergo randomisation. A subset of 155 patients were further studied on why randomisation did not proceed, and found that $56.0 \%$ were lost to randomisation due to clinical decisions from surgeons, oncologists and multidisciplinary tumour boards (MDT). In this trial, the main difficulty in recruitment was getting the clinician to relay the uncertainty of outcomes after LUM to both the patients and MDT, eventually succumbing to selection biases.

In addition, the confirmation of PM is tricky. Biopsy of pulmonary lesions can be challenging and are limited by its location, as well as its associated procedural risks. Diagnosis is henceforth made based on imaging findings, either at one sitting or over time. But, other differential diagnoses for such lung nodules may also include those of granulomas, primary lung cancers or other benign pathologies.

\section{CONCLUSION}

The benefit of LUM in metastatic CRC has not been well proven, despite being commonly performed. Our review shows that LUM failed to show any clear survival benefit when comparing LUM to LIM, and statistically non-significant marginal benefits when comparing to no surgery. We also showed that patients who underwent LUM in the included studies were likely to have a single tumour and have metachronous disease, highlighting the likelihood of selection biases in patient selection for LUM. Given the evidence available at present, we conclude that further prospective studies are needed to understand the true effect of LUM on outcomes in metastatic CRC.

\section{REFERENCES}

1. Misiakos EP, Karidis NP, Kouraklis G. Current treatment for colorectal liver metastases. World J Gastroenterol 2011;17:4067-75.

2. Vauthey J-N, Zorzi D, Pawlik TM. Making unresectable hepatic colorectal metastases resectable-does it work? Semin Oncol 2005;32:118-22.

3. Artigas V, Marín-Hargreaves G, Marcuello E, et al. [Surgical resection of liver metastases from colorectal carcinoma. Experience in Sant Pau Hospital]. Cir Esp (Engl Ed) 2007;81:339-44.

4. Weiser MR, Jarnagin WR, Saltz LB. Colorectal cancer patients with oligometastatic liver disease: what is the optimal approach? Oncology 2013;27:1074-8.

5. Mohammad WM, Balaa FK. Surgical management of colorectal liver metastases. Clin Colon Rectal Surg 2009;22:225-32.

6. Chakedis J, Schmidt CR. Surgical treatment of metastatic colorectal cancer. Surg Oncol Clin N Am 2018;27:377-99.

7. Mitry E, Guiu B, Cosconea S, et al. Epidemiology, management and prognosis of colorectal cancer with lung metastases: a 30-year population-based study. Gut 2010;59:1383-8.

8. Kanemitsu Y, Kato T, Hirai T, et al. Preoperative probability model for predicting overall survival after resection of pulmonary metastases from colorectal cancer. Br J Surg 2004;91:112-20.

9. Joanna Briggs Institute. Critical appraisal tools. Available at: https://jbi.global/critical-appraisal-tools. Accessed on 12 June 2021. 
10. Treasure T, Farewell V, Macbeth F, et al. Pulmonary metastasectomy versus continued active monitoring in colorectal cancer (PulMiCC): a multicentre randomised clinical trial. Trials 2019;20:718.

11. Milosevic M, Edwards J, Tsang D, et al. Pulmonary metastasectomy in colorectal cancer: updated analysis of 93 randomized patients control survival is much better than previously assumed. Colorectal Dis 2020;22:1314-24.

12. Zong Z, Zhou TC, Tang FX, et al. Impact of site-specific metastases on surgical value and survival among metastatic colorectal cancer patients. Am Surg 2020;86:220-7.

13. Socola F, Nguyen DM, Ochoa RE, et al. A cohort study evaluating the role of surgery for lung metastases from colorectal cancer. Anticancer Res 2015;35:3431-5.

14. Lee-Ying R, Bernard B, Gresham G, et al. A comparison of survival by site of metastatic resection in metastatic colorectal cancer. Clin Colorectal Cancer 2017;16:e23-8.

15. Iwasaki A, Shirakusa T, Yamashita Y, et al. Characteristic differences between patients who have undergone surgical treatment for lung metastasis or hepatic metastasis from colorectal cancer. Thorac Cardiovasc Surg 2005;53:358-64.

16. Gonzalez M, Poncet A, Combescure C, et al. Risk factors for survival after lung metastasectomy in colorectal cancer patients: a systematic review and meta-analysis. Ann Surg Oncol 2013; 20:572-9.

17. Girard P, Ducreux M, Baldeyrou P, et al. Surgery for lung metastases from colorectal cancer: analysis of prognostic factors. J Clin Oncol 1996;14:2047-53.

18. Tomlinson JS, Jarnagin WR, DeMatteo RP, et al. Actual 10-year survival after resection of colorectal liver metastases defines cure. J Clin Oncol 2007;25:4575-80.

19. Tampellini M, Ottone A, Bellini E, et al. The role of lung metastasis resection in improving outcome of colorectal cancer patients: results from a large retrospective study. Oncologist 2012;17:1430-8.

20. Sakamoto T, Tsubota N, Iwanaga $\mathrm{K}$, et al. Pulmonary resection for metastases from colorectal cancer. Chest 2001;119:1069-72.

21. Aberg T. Selection mechanisms as major determinants of survival after pulmonary metastasectomy. Ann Thorac Surg 1997;63:611-2. 